\title{
A PERMANENT-MAGNET FOCUSED X-BAND PHOTOINJECTOR
}

\author{
D. Yu, D. Newsham, P. Wilson, J. Zeng, DULY Research Inc. \\ J. Rosenzweig, X. Ding, UCLA \\ F. Hartemann, E. Landahl, ILSA/UCD
}

\section{Abstract}

A Plane-Wave-Transformer (PWT), integrated photoinjector operating at an $\mathrm{X}$-band frequency $(8.547 \mathrm{GHz})$ is being developed by DULY Research Inc. in a DOE SBIR project, in collaboration with UCLA and UCD/ILSA. Upward frequency scaling from an S-band PWT photoinjector would result in a compact photoinjector with unprecedented brightness. Challenging technological innovations are required at X-band. In particular, water cooling capacity, mechanical support strength, and materials properties do not scale linearly with frequency. Instead of using large solenoids, we have successfully designed the required focusing for an X-band PWT using a compact, permanent magnet system. Also described in this paper is a system design of the $\mathrm{X}$-band photoinjector, including the RF system and the cooling/support of the PWT structure.

\section{INTRODUCTION}

This paper reports an ongoing research project in which DULY Research Inc. develops a compact, highbrightness, $8.547-\mathrm{GHz}, 20-\mathrm{MeV}$ photoinjector using a plane-wave-transformer (PWT) design for the standingwave accelerating structure (Figure 1). The motivation for a high-frequency photoinjector lies in the great enhancement of beam brightness in a much smaller footprint, important for many commercial applications. Our design directly integrates the photocathode into a PWT linac. The integrated X-band PWT photoinjector has a designed beam brightness of $10^{15} \mathrm{~A} / \mathrm{m}^{2}$, an order of magnitude higher than an earlier S-band version, being constructed and now near completion in a DULY/UCLA collaboration $[1,2]$.

\section{BEAM BRIGHTNESS SCALING}

The beam brightness, by natural frequency scaling, is defined as:

$$
B \equiv 2 I / \varepsilon^{2} \propto Q / \sigma_{z} \varepsilon^{2} \propto \lambda^{-2},
$$

where $I$ is the beam current, or charge $(\mathrm{Q})$ per bunch per unit time, $\varepsilon$ is the emittance, $\sigma_{z}$ is the rms bunch length, and $\lambda$ is the RF wavelength. It is apparent from this expression that there is much to be gained by operating $\mathrm{RF}$ photoinjectors at high frequency. One can also show that including emittance dilution due to space charge, RF chromatic contribution and other field asymmetry effects, and keeping the charge density constant, the beam brightness for an X-band photoinjector is better than that achieved at S-band by roughly an order of magnitude.

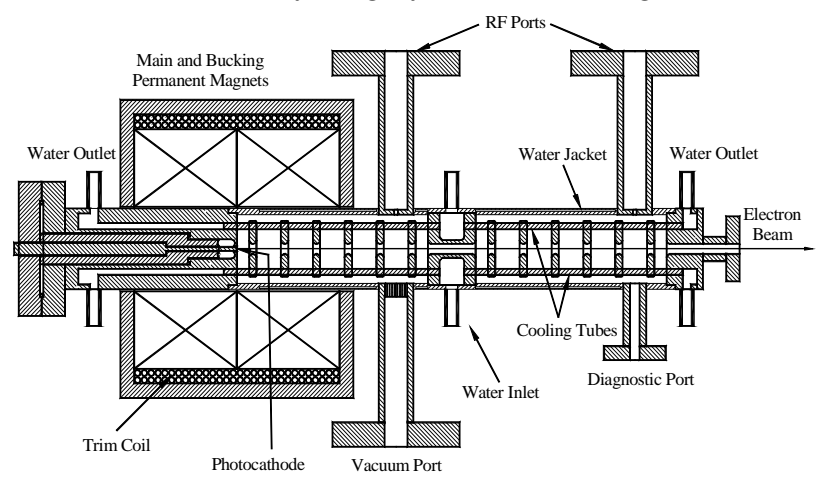

Figure 1: X-band PWT photoinjector schematic.

\section{PERMANENT MAGNET FOCUSING SYSTEM}

A prescribed magnetic field profile, following the principle of emittance compensation [3], is a key ingredient of success for the focusing and propagation of a small electron beam through the X-band PWT linac. The longitudinal magnetic field on axis must vanish at the photocathode, rise sharply to 3-4 kG in the first full cell or thereabouts, and then taper down to zero in a few more cells. The radial and azimuthal components of the magnet fields should be small. Such a magnetic field configuration assures not only that the beam emittance is preserved for the entire length of the accelerator, but also that the beam can be focused beyond to a spot sufficiently far away, where the first set of quadrupole magnets are located. Using solenoids to obtain the necessary magnetic field profile for the X-band PWT would require large

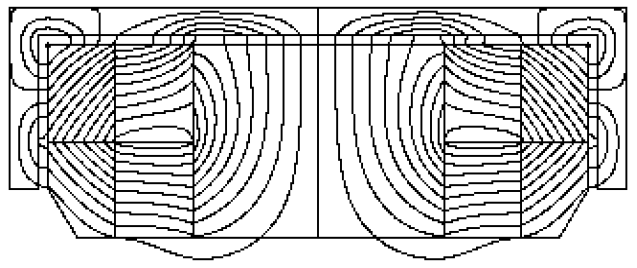

Figure 2: Field lines of permanent magnet.

coils, leaving little room for other essential, auxiliary structures of the linac such as the RF and vacuum ports.

DULY Research has proposed, and designed a hybrid, permanent magnet focusing system using the POISSON and PANDIRA codes. Figures 2 and 3 show the field profile due to the focusing magnets. The main and bucking magnets in this system are identical, but with 


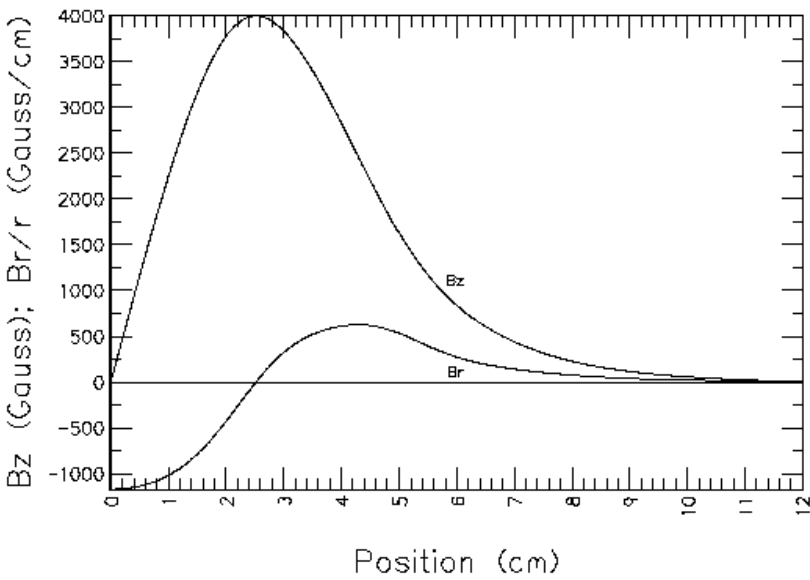

Figure 3: Field strength of permanent magnet.

opposite polarizations. An 80-turns, one-eighth-inch square trim coil is included for final adjustment of the null position of the longitudinal magnetic field at the cathode. A small current of 50A (or 4000 Ampere-turns) in the trim coil can move the axial position of the magnetic null by $1.4 \mathrm{~mm}$. The footprint of this system is quite small (about 6" diameter), and fits well over the PWT linac, as shown in Figure 1.

\section{RF SYSTEMS}

The main RF power supply for the X-band photoinjector linac will be a $8.547 \mathrm{GHz}$, SL3 klystron at ILSA/UCD which produces square RF pulses at power levels in excess of $15 \mathrm{MW}$ at $30 \mathrm{~Hz}$ repetition rate, with a pulse duration of $2 \mu \mathrm{s}$, and an amplitude ripple $<2 \%$. The phase stability of the klystron is currently measured at $\pm 10^{\circ}$ over the pulse duration, and will be brought down to $\pm 1^{\circ}$ by using a phase stabilisation feedback loop. The 2$\mathrm{kW}$ TWTA input drive to the klystron will be synchronised to the laser oscillator, using a phase-locked dielectric resonance oscillator to upconvert the laser oscillator output frequency to the desired X-band drive frequency.

We have designed a RF system using the SL3 klystron power supply to provide sufficient energy to accelerate an electron beam in the X-band PWT photoinjector to $20 \mathrm{MeV}$. Since the PWT is a standing-wave structure, some RF power may be reflected during startup and conditioning. To prevent the reflected power from damaging the klystron, we use a $3 \mathrm{~dB}$ power splitter to divide the main RF into two feeds with $90^{\circ}$ phase difference (Figure 4a). The PWT linac structure is also split, and the two linac sections are fed by the two feeds from the power splitter. Thus, no reflected power will get back to the klystron (assuming the two linac sections have the same coupling coefficient and are both tuned to resonance); and no high-power isolator is needed.

Because the SL3 klystron RF pulse is long $(2 \mu \mathrm{s})$ compared with the filling time of the PWT linac (292 ns), a SLED pulse compression system [4] may be optionally installed between the klystron and the aforementioned power splitter (Figure 4b) to increase the linac energy gain. SLED stores some of the pulse energy, which would otherwise be wasted, and delivers it to the structure. Calculations show that an additional 30\% energy gain for the electrons in the standing-wave PWT linac may be achieved with SLED.
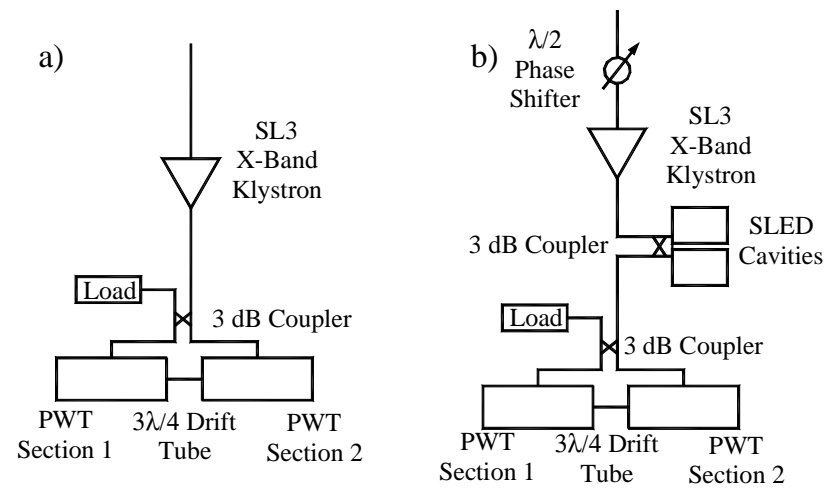

Figure 4: a) Schematic for RF feed to X-band PWT.

b) Same system with SLED pulse compression.

\section{PWT ACCELERATING STRUCTURE}

To compensate for the phase difference between the feeds, the two linac sections are connected by a short drift tube having a length equal to $3 \lambda / 4$. In this case, the RF phase of the second section is ahead of that of the first section by $90^{\circ}$. The photocathode is inserted through a demountable flange and integrated into the center of the end plate of the first PWT linac section. The linac consists of a series of suspended disks which are supported and cooled by water-carrying tubes. Two inlets, through the center divider of the two linac sections, feed water into eight tubes in parallel, four in each section; and the water outlets are located outside the end plates at the far ends of the linac sections. No internal cooling channels inside the disks are needed.

The RF properties for the X-band PWT linac are calculated using the 3D electromagnetic code GdfidL, and are shown in Table 1 for the accelerating mode, for the cases with 1) no cooling rod, 2) four cooling rods with a diameter of $0.83 "$, scaled from the S-band version, and 3) four rods with a larger diameter of 0.125". The RF degradation for the case with larger rods is not overwhelming compared with the smaller rods. The case with no rods has the best RF properties. This can be implemented with disks supported by synthetic diamond washers as proposed by DULY Research [5].

The X-band PWT linac has a good frequency separation between the accelerating mode and the nearest 0-mode, i.e. $1043 \mathrm{MHz}$ and $612 \mathrm{MHz}$, respectively, for the configurations with four thin and thick rods. The dipole and quadrupole modes are insignificant for these cases.

Based on the available klystron power and the RF properties of the linac, the structural parameters, expected 
accelerating gradients, and energy gains are shown in Table 3, for several RF/linac configurations (Table 2). The variations are the length of the linac, and whether or not a SLED pulse compression is used. We have used the values of $\mathrm{Q}, \mathrm{r} / \mathrm{Q}$ and $\mathrm{r}$ of the 4-thin-rod PWT linac (Table 1) to calculate the linac parameters shown in Table 3. Using larger rods (0.125" diameter), the shunt impedances are lower by 6-7\%, and the corresponding energy gains and gradients by only 3-4\%. Including waveguide losses (about $1.5 \% / \mathrm{m}$ at $8.5 \mathrm{GHz}$ ) the actual energy gains and gradients may be about $10 \%$ less than the values shown on Table 3 .

Table 1: RF Properties of $\pi$-Mode for the PWT Linac

\begin{tabular}{|l|l|l|l|}
\hline & $\mathrm{r} / \mathrm{Q}(\Omega / \mathrm{m})$ & $\mathrm{Q}$ & $\mathrm{r}(\mathrm{M} \Omega / \mathrm{m})$ \\
\hline No Rod & 6,488 & 21,657 & 140.5 \\
\hline 4 Thin Rods & 7,578 & 15,717 & 119.1 \\
\hline 4 Thick Rods & 8,510 & 13,052 & 111.1 \\
\hline
\end{tabular}

Table 2: X-Band Linac Configurations

\begin{tabular}{|l|l|l|l|}
\hline Case & $\begin{array}{l}\text { RF Pulse } \\
\text { Compression }\end{array}$ & $\begin{array}{l}\text { No. of } \\
\text { Sections }\end{array}$ & $\begin{array}{l}\text { No. of cells in each } \\
\text { section }\end{array}$ \\
\hline 1 & None & 2 & 5 full +2 half \\
\hline 2 & None & 2 & 7 full +2 half \\
\hline 3 & SLED & 2 & 5 full +2 half \\
\hline 4 & SLED & 2 & 7 full +2 half \\
\hline
\end{tabular}

Table 3: X-Band PWT Linac Parameters

\begin{tabular}{|l|l|l|l|l|}
\hline & Case 1 & Case 2 & $\begin{array}{l}\text { Case 3 } \\
\text { (SLED) }\end{array}$ & $\begin{array}{l}\text { Case 4 } \\
\text { (SLED) }\end{array}$ \\
\hline Section length $(\mathrm{cm})$ & 10.53 & 14.04 & 10.53 & 14.04 \\
\hline Cells per section & $5+2 / 2$ & $7+2 / 2$ & $5+2 / 2$ & $7+2 / 2$ \\
\hline Filling time $(\mathrm{ns})$ & 293 & 293 & 167 & 167 \\
\hline Shunt impedance $(\mathrm{M} \Omega)$ & 12.54 & 16.72 & 12.54 & 16.72 \\
\hline Energy gain/section $(\mathrm{MeV})$ & 9.70 & 11.20 & 12.61 & 14.56 \\
\hline Final energy $(\mathrm{MeV})$ & 19.40 & 22.40 & 25.22 & 29.12 \\
\hline Linac length $(\mathrm{cm})$ & 21.06 & 28.08 & 21.06 & 28.08 \\
\hline Gradient $(\mathrm{MV} / \mathrm{m})$ & 92.10 & 79.76 & 119.7 & 103.6 \\
\hline
\end{tabular}

\section{BEAM DYNAMICS SIMULATIONS}

We have performed simulations of the split injector configuration for Case 1 ( 2 sections, each having 5 full + 2 half cells), and Case 2 (2 sections, each having 7 full + 2 half cells). The PARMELA simulations use as input files the full magnetic field profile (longitudinal and transverse), obtained with POISSON and PANDIRA, and the electrical field profile, with SUPERFISH. The beam charge, spot size and bunch length, as well as the computed emittances and rms radius for the two cases are summarized in Table 4 and Figures $5 \mathrm{a}-\mathrm{d}$. Both cases yield a high beam brightness $\left(0.8 \times 10^{15} \mathrm{~A} / \mathrm{m}^{2}\right)$, and the longer structure (Case 2) results in a higher energy gain, as expected. The peak gradients shown in Table 4 are consistent with the available RF power. The average gradients are $95 \mathrm{MV} / \mathrm{m}$ and $82 \mathrm{MV} / \mathrm{m}$, for cases 1 and 2 . The normalized beam emittance ( $1 \mathrm{~mm}-\mathrm{mrad})$ and energy spread $(0.2 \%)$ are low, proving the validity of the principle of emittance compensation in the X-band PWT linac. Effects of the RF octupole due to the rods on the accelerating mode have been estimated in a companion PAC99 paper [6] and are small for charge less than $2 \mathrm{nC}$.
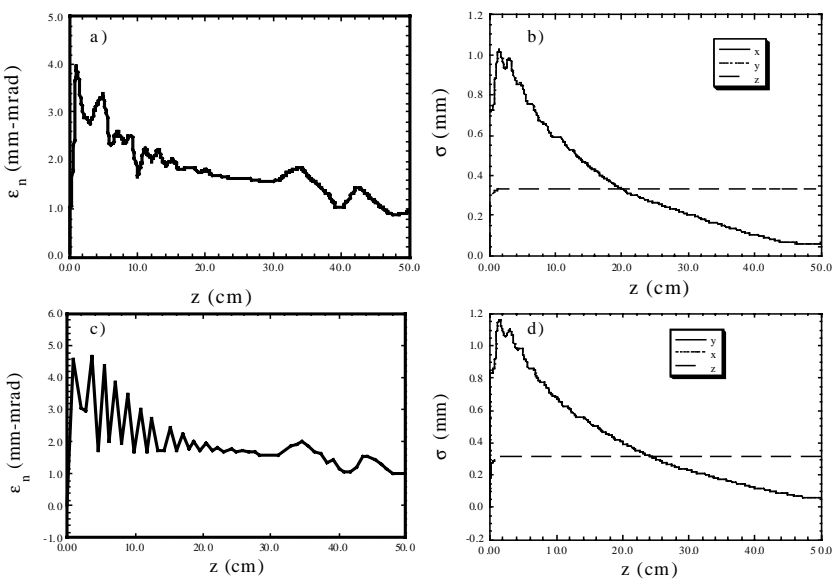

Figure 5: Results of PARMELA simulations. a) and b) are emittance and rms radius for Case 1. c) and d) are emittance and rms radius for Case 2.

Table 4: Summary of PARMELA Calculations

\begin{tabular}{|l|l|l|}
\hline & Case 1 & Case 2 \\
\hline Active linac length (cm) & 21 & 28 \\
\hline Number of cells & $2 \times(5+2 / 2)$ & $2 \times(7+2 / 2)$ \\
\hline Peak magnetic field (Gauss) & 3315 & 2965 \\
\hline Charge per bunch (nC) & 1.0 & 1.0 \\
\hline Bunch Length (mm) & 0.36 & 0.33 \\
\hline $\begin{array}{l}\text { RMS transverse beam size (at } \\
\text { cathode) }(\mathrm{mm})\end{array}$ & 0.70 & 0.84 \\
\hline Emittance $(\mathrm{mm}-\mathrm{mrad})$ & 0.95 & 1.00 \\
\hline Energy spread $(\%)$ & 0.2 & 0.2 \\
\hline Final Energy $(\mathrm{MeV})$ & 20 & 23 \\
\hline Peak gradient $\left(\mathrm{MV} / \mathrm{m}^{2}\right)$ & 156 & 180 \\
\hline Beam brightness $\left(\mathrm{A} / \mathrm{cm}^{2}\right)$ & $0.8 \times 10^{15}$ & $0.8 \times 10^{15}$ \\
\hline
\end{tabular}

\section{CONCLUSIONS}

We have demonstrated the feasibility of a highbrightness, X-band photoinjector by design and simulations. In the next phase of project, we will fabricate and test the X-band photoinjector upon DOE approval.

"Work supported by DOE SBIR DE-FG03-98ER82566.
" Email: duly@ technologist.com

[1] D. Yu et al.,. Proc. of Particle Accelerator Conf., Vancouver, B.C. Canada, May 1997, p. 2802.

[2] X. Ding et al., these proceedings.

[3] L. Serafini and J. Rosenzweig, Phys. Rev. E, 55, 6B, p.7565-90, 1997. and B.E. Carlsten, Proc. of Particle Accelerator Conf., Chicago, IL, March 1989, p. 313.

[4] Z.D. Farkas, H.A. Hogg, G.A. Loew, P.B. Wilson, SLACPUB-1453, June 1974.

[5] D. Yu and T. Lee, Patent pending.

[6] J. Rosenzweig, S. Anderson, X. Ding, D. Yu., these proceedings. 